\title{
High dosage of zinc modulates T-cells in a time-dependent manner within porcine gut-associated lymphatic tissue
}

\author{
Susanne Kreuzer-Redmer ${ }^{1,2} \dagger^{*}$, Danny Arends ${ }^{1} \dagger$, Jasper N. Schulte ${ }^{1}$, Diana Karweina ${ }^{1}$, Paula Korkuc ${ }^{1}$, \\ Nadine Wöltje ${ }^{1}$, Deike Hesse ${ }^{1}$, Robert Pieper ${ }^{3}$, Volker Gerdts ${ }^{4}$, Jürgen Zentek ${ }^{3}$, Francois Meurens ${ }^{5}$ and \\ Gudrun A. Brockmann ${ }^{1}$ \\ ${ }^{1}$ Breeding Biology and Molecular Genetics, Thaer-Institut, Humboldt-Universität zu Berlin, Invalidenstrasse 42, \\ 10115 Berlin, Germany \\ ${ }^{2}$ Department for Farm Animals and Veterinary Public Health, Institute of Animal Nutrition and Functional Plant \\ Compounds, University of Veterinary Medicine Vienna, 1210 Vienna, Austria \\ ${ }^{3}$ Institute of Animal Nutrition, Freie Universität Berlin, Königin-Luise-Str. 49, 14195 Berlin, Germany \\ ${ }^{4}$ Vaccine and Infectious Disease Organization and Department of Veterinary Microbiology, Western College of Veterinary \\ Medicine, University of Saskatchewan, Saskatoon, SK, Canada S7N5E3 \\ ${ }^{5}$ BIOEPAR, INRA, Oniris - Nantes Atlantic National College of Veterinary Medicine, La Chantrerie, 44307 Nantes, France \\ (Submitted 14 February 2018 - Final revision received 1 August 2018 - Accepted 12 September 2018)
}

\section{Abstract}

Zn serves as a powerful feed additive to reduce post-weaning diarrhoea in pigs. However, the mechanisms responsible for Zn-associated effects on the adaptive immune responses following feeding of a very high dosage of $\mathrm{Zn}$ remain elusive. In this study, we examined the $\mathrm{T}$-cell response in gut-associated lymphatic tissues of seventy-two weaned piglets. Piglets received diets with $57 \mathrm{mg} \mathrm{Zn/kg} \mathrm{(low} \mathrm{Zn} \mathrm{concentration,}$ LZn), $164 \mathrm{mg} \mathrm{Zn/kg} \mathrm{(medium} \mathrm{Zn} \mathrm{concentration,} \mathrm{MZn)} \mathrm{or} 2425 \mathrm{mg} \mathrm{Zn} / \mathrm{kg}$ (high Zn concentration, HZn) mg Zn/kg feed for 1,2 or 4 weeks. We observed that feeding the HZn diet for 1 week increased the level of activated T-helper cells (CD $4^{+}$and CD $8 \alpha^{\mathrm{dim}}$ ) compared with feeding MZn and LZn $(P<0.05)$. In addition, we observed higher transcript amounts of interferon $\gamma$ and T-box 21 (TBET) in the HZn group compared with the MZn and LZn groups $(P<0 \cdot 05)$. A gene set enrichment analysis revealed an over-representation of genes associated with 'cytokine signalling in immune system'. Remarkably, feeding of a very high $\mathrm{Zn}$ dosage led to a switch in the immune response after 2 weeks. We detected higher relative cell counts of $\mathrm{CD}^{+}{ }^{+} \mathrm{CD} 25^{\text {high }}$ regulatory T-helper cells $(P<0.05)$ and a higher expression of forkhead box P3 (FOXP3) transcripts $(P<0 \cdot 05)$. After 4 weeks of feeding a high-dosage $\mathrm{Zn}$ diet, the relative $\mathrm{CD} 4^{+} \mathrm{T}$-cell count $(P<0 \cdot 05)$ and the relative $\mathrm{CD} 8 \beta^{+} \mathrm{T}$-cell count $(P<0 \cdot 1)$ were reduced compared with the MZn group. We hypothesise that after 1 week the cellular T-helper 1 response is switched on and after 2 weeks it is switched off, leading to decreased numbers of T-cells.

Key words: Zinc: Nutritional immune response: T-cells: Pigs

The micronutrient $\mathrm{Zn}$ is an essential trace element that is required for physical growth, development and immune defence $^{(1)}$. A lack of $\mathrm{Zn}$ can lead to growth and developmental retardation, an impairment of the immune system together with decreased resistance to infections and therefore can cause diverse diseases in humans and animals ${ }^{(2)}$. Several disorders related to $\mathrm{Zn}$ deficiency such as diarrhoea have been shown to be recovered and prevented by $\mathrm{Zn}$ supplementation as adjunct therapy $^{(3)}$. Different studies demonstrate that $\mathrm{Zn}$ deficiency impairs the formation and activity of inflammatory cytokines and therefore has an influence on the development and regulation of immune cells ${ }^{(4,5)}$. A deficit of $\mathrm{Zn}$ can thus alter the immune response, resulting in an ineffective control of pathogens ${ }^{(6)}$.

However, further investigations are needed to fully understand the precise effects of $\mathrm{Zn}$ on the immune system. In particular, the pig, which is known to have a digestive physiology similar to humans as it is an omnivore and the organ size is comparable to humans, could be used as a translational $\operatorname{model}^{(7,8)}$.

\footnotetext{
Abbreviations: BW, body weight; FOXP3, forkhead box P3; GALT, gut-associated lymphatic tissue; GATA3, GATA binding protein 3; GSEA, gene set enrichment analysis; HZn, high Zn concentration; IFN , interferon $\gamma$; LZn, low Zn concentration; MLN, mesenteric lymph node; MZn, medium Zn concentration; TBET, T-box 21; ZIP4, solute carrier family 39 member 4 (SLC39A4); ZnT, solute carrier family 30 (SLC30).
}

* Corresponding author: S. Kreuzer-Redmer, email Susanne.Kreuzer.1@agrar.hu-berlin.de

$\dagger$ Contributed equally to this work. 
In pig husbandry, $\mathrm{Zn}$ is known to induce several positive effects on the gastrointestinal microbiota when fed at a high dietary level (2000-3000 parts per million (ppm)), including enhanced growth of the pigs, and shows improved resistance against post-weaning diarrhoea ${ }^{(9-12)}$.

Despite its positive effects, there are also negative aspects of $\mathrm{Zn}$ that need to be considered. Several lines of evidence suggest that long-term uptake of high dosage of $\mathrm{Zn}$ can lead to negative effects on the immune system. Previous studies using an infection model with Salmonella typhimurium revealed that long-term application of zinc oxide over a period of 6 weeks led to decreased number of T-cell populations within the mesenteric lymph nodes (MLN) of the pigs ${ }^{(13)}$. Within the same experimental setup, we observed increased expression of suppressor of cytokine signalling (SOCS) SOCS2, SOCS4 and SOCS7 in MLN of piglets, which suggested that such treatments may impair the immune response ${ }^{(14)}$. These results are consistent with previous findings that permanently high uptake of $\mathrm{Zn}$ can result in an impairment of lymphocyte proliferation $^{(15)}$. Moreover, there is some evidence for elevated levels of liver enzymes associated with liver damage in pigs ${ }^{(16)}$ supplemented with high level of $\mathrm{Zn}$, and co-accumulation of $\mathrm{Cu}$ and $\mathrm{Zn}$ was found in the kidney of piglets fed very high concentrations of zinc oxide for 4 weeks ${ }^{(17)}$. It was shown that the $\mathrm{CD}^{+} \gamma \delta$ T-cell fraction of intra-epithelial lymphocytes showed reduced abundance after 4 weeks of feeding a high $\mathrm{Zn}$ diet compared with the control diet ${ }^{(18)}$. However, a more detailed analysis of the immune-regulating genes providing insight into beneficial and impairing mechanisms of the T-cell immune response within the gut-associated lymphatic tissues (GALT) after feeding high $\mathrm{Zn}$ dosages is still missing.

Our aims are to investigate (1) whether $\mathrm{Zn}$ transporters are expressed on immune cells; (2) whether the adaptive immune cells of the GALT respond to, and are affected by, feeding of Zn in a dosage-dependent manner and (3) how GALT is influenced by the exposure duration to different dosages of $\mathrm{Zn}$.

Therefore, we examined the relative counts of T-cells and the relative expressions of $\mathrm{Zn}$ transporters and immune-relevant genes in GALT of seventy-two weaned piglets fed different levels of $Z n$ at 1, 2 or 4 weeks. We aimed to use a low $Z n$ concentration (LZn) of $50 \mathrm{mg} \mathrm{Zn/kg} \mathrm{feed,} \mathrm{which} \mathrm{should} \mathrm{mimic} \mathrm{a} \mathrm{suboptimal} \mathrm{Zn}$ status for the piglets, a medium $\mathrm{Zn}$ concentration (MZn) of $150 \mathrm{mg} \mathrm{Zn} / \mathrm{kg}$ feed and a very high $\mathrm{Zn}$ concentration (HZn) of $2500 \mathrm{mg} \mathrm{Zn} / \mathrm{kg}$ feed. Feed with HZn of $2250-3000 \mathrm{mg} \mathrm{Zn} / \mathrm{kg}$ is commonly used as medical intervention to treat post-weaning diarrhoea ${ }^{(14,19-21)}$. The MZn group was our control group, as the European Commission has restricted dietary supplementation of $\mathrm{Zn}$ to a maximum of $150 \mathrm{ppm}$ for piglets. Therefore, $150 \mathrm{ppm}$ is the commonly used dosage in feed in Europe. In addition, we used the LZn group to reflect the effects of short-term subclinical Zn deficiency ${ }^{(22)}$ as a counterpart to the HZn group.

\section{Methods}

\section{Animals, housing and diets}

A total of seventy-two purebred Landrace piglets were weaned at 26 (sD 1) d of age with a mean body weight (BW) of $7 \cdot 2$
(SD 1.2 ) $\mathrm{kg}$ and randomly allocated into three dietary treatment groups balancing for sex, litter and BW. BW and lymphoid tissue sizes can be found in online Supplementary Table S1; pairwise $t$ tests were performed between the three feeding groups to detect difference in relative lymphoid tissue size between the groups (no significant differences were detected; online Supplementary Table S1 - sheet 4). Animals were housed in pens ( $n 2$ per pen) with straw bedding and ad libitum access to feed and water. Each dietary group ( $n 24$ per feeding group) was fed a common basal maize-wheat-barleysoyabean diet with different $\mathrm{Zn}$ levels (online Supplementary Table S2) ${ }^{(23)}$. The dietary $\mathrm{Zn}$ levels were confirmed by analysis using atomic absorption spectrometry in an AAS vario 6 spectrometer (Analytik Jena). We aimed to have 50, 150 or $2500 \mathrm{mg} \mathrm{Zn}$ in $1 \mathrm{~kg}$ of feed; the actual measured values for the feedings groups were 57 (LZn), 164 (MZn) or 2425 (HZn) mg $\mathrm{Zn} / \mathrm{kg}$. The Zn source was analytical-grade zinc oxide (Sigma Adrich). No antibiotics were administered before and during the experiment. All animal experiments were performed at the Institute of Animal Nutrition, Freie Universität, Berlin, Germany, in accordance with the German Animal Protection Law and approved by the local responsible authorities (LaGeSo Reg. No. 0347/09). The in vivo procedures were consistent and in compliance with the Animal Research: Reporting of In Vivo Experiments (ARRIVE) Guidelines for Reporting Animal Research.

\section{Sampling of gut-associated lymphatic tissue and immune cells}

In all, eight piglets per group and time point were euthanised at the following time points: 32 (SD 1), 39 (SD 1) and 53 (SD 1) d of age. The piglets were sedated with $20 \mathrm{mg} / \mathrm{kg} \mathrm{BW}$ of ketamine hydrochloride (Ursotamin ${ }^{\circledR}$; Serumwerk Bernburg AG) and $2 \mathrm{mg} / \mathrm{kg} \mathrm{BW}$ of azaperone (Stresnil ${ }^{\circledR}$; Jansen-Cilag), and cord blood was taken before euthanasia with intra-cardial injection of $10 \mathrm{mg} / \mathrm{kg}$ BW of tetracaine hydrochloride, mebezonium iodide and embutramide (T61 ${ }^{\circledR}$; Intervet). During necropsy, jejunal and ileocaecal mesenteric lymph nodes (JELN and ILLN, respectively) and Peyer's patches from the jejunum and the ileum (JEPP and ILPP, respectively), as well as the ileal papilla (PAPIL), were obtained and either frozen in liquid $\mathrm{N}_{2}$ within 3 min after death of the animal or collected in $15-\mathrm{ml}$ Falcon tubes filled with $5 \mathrm{ml}$ of PBS containing $0.2 \%$ bovine serum albumin. Lymphocytes were isolated using a $70-\mu \mathrm{m}$ Cell Strainer (Becton Dickinson GmbH) as described before ${ }^{(24)}$ and purified by centrifugation in a Ficoll gradient.

\section{Zinc concentration in mesenteric lymph nodes}

MLN were freeze-dried before hydrolysis in hydrochloric acid and subsequently analysed by atomic absorption spectrometry in an AAS vario 6 spectrometer. An external standard (PerkinElmer Pure XVI, \#N9300281; PerkinElmer) was used for calibration. The accuracy was checked by a certified reference $\mathrm{Zn}$ solution (Titrisol, \#9953; Merck). 
Cell culture of isolated leucocytes from mesenteric lymph nodes

As direct effects of $\mathrm{Zn}$ on the immune cells are difficult to study in vivo, owing to many environmental influencers of immune status, we opted for a cell culture approach using primary isolated immune cells. We believe that this approach should lead to reduced complexity of the studied system compared with in vivo experiments. However, the use of primary isolated immune cells should give results reflecting the interplay of different immune cells more accurately compared with using single-origin cell lines. Cells were isolated from one healthy control piglet; $1 \times 10^{6}$ cells/well were cultured in a ninety-sixwell plate using Roswell Park Memorial Institute (RPMI) media enriched with $10 \%$ fetal calf serum, $200 \mathrm{~mm}$ L-glutamine and Antibiotic-Antimycotic (Thermo Fisher Scientific). The aim of the in vitro experiments was to mimic the same concentrations of $\mathrm{Zn}$ in cell culture as they occur in vivo. The analyses of $\mathrm{Zn}$ concentrations in MLN revealed on average $13 \mathrm{mg} \mathrm{Zn} / \mathrm{kg}$. If one accounts for the molarity of $\mathrm{Zn}$, this calculates $0.2 \mathrm{~mm} \mathrm{Zn/litre} \mathrm{in}$ the cell medium to reflect the in vivo measured concentration. However, this is not the concentration in the cell. In preexperiments, we tested $\mathrm{Zn}$ concentrations from $10 \mu \mathrm{m}$ to $2 \mathrm{~mm}$ from different organic and inorganic $\mathrm{Zn}$ sources in porcine and murine splenocytes and lymphocytes from MLN. Although $\mathrm{ZnSO}_{4}$ and zinc oxide were toxic to the primary cultured cells in the used concentrations of 100 or $200 \mu \mathrm{M} \mathrm{Zn}^{2+}$, the cells survived for $5 \mathrm{~d}$ when the medium was supplemented with $200 \mu \mathrm{M}$ zinc gluconate or zinc acetate per litre. For our experiments, we chose to add zinc acetate and we calculated the $\mathrm{Zn}^{2+}$ amount to be 10,100 or $200 \mu \mathrm{m} \mathrm{Zn}^{2+}$ per well to reflect low and high levels of $\mathrm{Zn}$ for the primary cultured leucocytes.

\section{Flow cytometry}

Flow cytometry (FCM) was performed with the purified lymphocytes from the intestinal tissues and blood. Combinations of surface antigens were used to detect the following cell types T-helper cells: CD4 ${ }^{+}$(Clone 74-12-4; Southern Biotech), CD25 $5^{+/-}$ (Clone K231.3B2; Biozol) and $\mathrm{CD} 8 \alpha^{-/ \mathrm{dim}}$ (Clone 76-2-11; Southern Biotech); regulatory T-cells: $\mathrm{CD}^{+}, \mathrm{CD} 25^{\text {high }}$; and cytotoxic T-cells: CD8 $\beta^{+}$(Clone: PG164A; VMRD) and CD $4^{-}$. For forty randomly chosen samples, we performed an additional intra-nuclear staining of forkhead box P3 (FOXP3) (Clone FJK-16s; eBioscience) to assess the regulatory T-cell phenotype as well. In these samples, the relative cell counts of $\mathrm{CD} 4^{+} \mathrm{CD} 25^{\text {high }}$ and $\mathrm{CD} 4^{+} \mathrm{FOXP} 3^{+}$were the same. For each reaction, $1 \times 10^{6}$ cells were stained as described elsewhere ${ }^{(25)}$. Per sample, 50000 lymphocytes, which were negative for propidium iodide staining $(0.5 \mu \mathrm{g} / \mathrm{ml})$, were assessed by FCM using a BD FACSCalibur ${ }^{\mathrm{TM}}$ flow cytometer (Becton Dickinson).

\section{Gene expression in tissue and isolated lymphocytes}

Tissue samples were homogenised by the FastPrep-24 Instrument (MP Biomedicals), and RNA was isolated using the NucleoSpin ${ }^{\circledR}$ RNA II Kit of Macherey-Nagel according to the manufacturer's instructions. The integrity of total RNA was measured by the
Agilent 2100 Bioanalyzer (Agilent Technologies). RNA samples with an RNA integrity number (RIN) value higher than 7.5 were taken for quantification. The quantity was assessed by a NanoDrop spectrophotometer (PEQLAB Biotechnologie GmbH). In all, $1 \mu \mathrm{g}$ of total RNA was reverse-transcribed into complementary DNA (cDNA) using AffinityScript quantitative PCR (qPCR) cDNA Synthesis Kit (Agilent) with oligo(dT) primers. Expression of $60 \mathrm{~S}$ ribosomal protein L19 (RPL19) and TATA box-binding protein $(T B P)$ did not vary owing to the $\mathrm{Zn}$ treatment of the piglets within the used tissues and cells and were therefore used as stable reference genes for normalisation of expression data for every sample on each plate. The primers and their source are described in online Supplementary Table S3. Primers for T-box 21 (TBET), FOXP3, GATA binding protein 3 (GATA3), interferon $\gamma(I F N \gamma)$, IL12 and protein kinase $\mathrm{C}$ theta (PKCtheta) as well as the solute carrier family 39 member 4 (SLC39A4; ZIP4) transcripts were designed with exon-boundaries overlapping, based on published sequences of the pig (Ensembl, Genome assembly: Sscrofa10.2) using the online primer design tool Primer3 (http://frodo.wi.mit. edu). Expression of the $\mathrm{Zn}$ transporter genes ZnT1, ZnT2 and ZnT5 (solute carrier family 30; SLC30) was determined for the principal isoform. The $\mathrm{Zn}$ transporter gene ZIP4 was examined for different protein-coding transcripts. The long transcripts ZIP4201 and ZIP4-001 contain all twelve exons of the ZIP4 gene; however, ZIP4-001 has a shortened exon 1 with a back-shifted transcription start site. The real-time qPCR (RT-qPCR) reaction was carried in a total volume of $10 \mu \mathrm{l}$ containing $6 \mu \mathrm{mol}$ forward primer, $6 \mu \mathrm{mol}$ reverse primer, $10 \mathrm{ng}$ of cDNA and $5 \mu \mathrm{l}$ of SYBR Select Master Mix (Applied Biosystems). The amplification protocol included a 10 -min denaturation step at $95^{\circ} \mathrm{C}$, followed by forty cycles consisting of $30 \mathrm{~s}$ at $95^{\circ} \mathrm{C}, 20 \mathrm{~s}$ at $60^{\circ} \mathrm{C}$ and $40 \mathrm{~s}$ at $72^{\circ} \mathrm{C}$. The quantification of the transcript amounts was performed with a ViiA ${ }^{\mathrm{TM}} 7$ Real-time PCR System (Applied Biosystems) or for the cell culture experiments with a Thermal Cycler (Bio-Rad). Afterwards, a melting curve analysis was performed in every reaction well to check for specificity of primers. All primer sets were initially validated for single amplicon generation, and standard curves were tested before analysis. Only primer pairs with efficiency rates between 90 and $110 \%$ were considered for gene expression analyses. Melting curves and PCR efficiency were used as standard quality criteria for each RT-qPCR run. Relative transcript amounts were calculated using the relative quantification method $\left(\Delta \Delta \mathrm{C}_{\mathrm{t}}\right)^{(26)}$. To calculate the $\Delta \mathrm{C}_{\mathrm{t}}$, the mean threshold cycle $\left(\mathrm{C}_{\mathrm{t}}\right)$ values of the two endogenous control genes RPL19 and TBP were subtracted from the $\mathrm{C}_{\mathrm{t}}$ of the target gene. To compare the relative expression among the different tissues and diets, the mean of the $\Delta \mathrm{C}_{\mathrm{t}}$ values of animals belonging to the MZn group (our control group) from the Peyer's patch were taken as the external calibrator to calculate the $\Delta \Delta \mathrm{C}_{\mathrm{t}}$. Results are presented as relative expression $=2^{-\Delta \Delta \mathrm{Ct}}$. Results shown for GALT represent the condensed analyses as means from separately analysed relative expressions from JELN and ILLN and JEPP and ILPP, as well as the PAPIL.

\section{RNA sequencing}

To get a deeper insight into the mechanism accounting for the effects of high dietary $\mathrm{Zn}$, we performed a deep sequencing 
approach for mRNA of MLN from 32-d-old piglets after feeding MZn or HZn in the feed for 1 week. Sequencing of individually barcoded samples was carried out for two male and one female piglet for MZn and HZn.

Read alignment. Paired-end sequencing reads were obtained using an Illumina instrument and were trimmed using trimmomatic 0.32 using the TruSeq3-PE-2.fa adapter library from Illumina. After trimming alignment was done by tophat 2 using the Sscrofa10.2 genome (Ensembl) and the Sscrofa10.2 transcriptome (Ensembl), we did not allow for multi-mapped reads (prefilter-multihits) and allowed for two mismatches, 13-bp alignment gaps and a maximum insertion and deletion size of $20 \mathrm{bp}$ when aligning sequences to the reference genome/transcriptome. After alignment reads were sorted using samtools (version 1.3.1-45) and optical duplicate were marked using picard tools (version 1.99), the resulting bamfiles were indexed using samtools, after which base recalibration around known SNP was performed using GATK (3.2-2) according to GATK best practices.

Gene expressions from RNA-sequencing. After alignment of reads, read-level reads per kilobase million (RPKM) gene expressions were performed using the following procedure: using the GenomicFeatures $\mathrm{R}$ package, we made a transcript database (exons by gene) from the Sus_scrofa.Sscrofa10.2.84. short.gtf file obtained from Ensembl. Read overlap between the genes and bamfiles was performed using the summarizeOverlaps function from the GenomeAlignments $\mathrm{R}$ package in union mode, ignoring strand orientation but allowing for fragments within a feature to be counted. Raw read counts were extracted and quantile normalised and transformed into RPKM values. These data were then log2-transformed to follow a normal distribution followed by differential expression analysis using standard two-sided $t$ tests. Genes were annotated using biomaRt, using the sscrofa_gene_ensembl database (online Supplementary Table S4).

Gene set enrichment analysis. We used the FGSEA package in $\mathrm{R}$ to perform gene set enrichment analysis (GSEA) on our differentially expressed genes. Therefore, we converted the Ensembl gene identifiers of our data into Entrez identifiers using the biomaRt $\mathrm{R}$ package. When a gene had no corresponding Entrez identifier in the biomaRt conversion, we used the Entrez identifier associated with the human orthologue for the corresponding gene. After converting identifiers, we then used the reactomePathways function provided by the FGSEA package to map pathways annotations to our Entrez identifiers. Settings used in the fgsea analysis function were 10000000 permutations; minimum size of the gene set to test was 80; and the maximum size of the gene set to test was 500. These parameters were chosen as we only want to look for over-representation in larger pathways as our sample size is low, and we expect a lot of false positives when performing GSEA using small pathways. Finally, we extracted over-represented pathways and plotted the figure using the topPathways function.

\section{Statistical analyses}

Statistical analyses of data. FCM raw data were analysed using FlowJo version 7.9 and further processed using $\mathrm{R}$ version 3.2.3. Plots were generated using the $\mathrm{R}$ package ggplot2. Zn group pairs (LZn against MZn and $H Z n$ against $M Z n$ ) were tested using pairwise Mann-Whitney $U$ tests. Multiple testing correction was not applied because of the small sample sizes. Relative gene expressions were calculated and analysed with Microsoft Excel 2010. Results were considered significant when $P \leq 0 \cdot 05$. The effect of $\mathrm{Zn}$ concentrations was tested using ANOVA (Table 1); as no significant differences were detected, a post hoc test was not performed.

Statistical power estimation. Estimation of differences in relative cell count, as well as in relative expression of genes, using a statistical power of $80 \%$ is performed using pairwise Whitney $U$ tests for the LZn and HZn dosage groups against the control MZn group. The minimally detectable effect size required was set to 1 unit of difference in relative expression level or relative cell count, leading to an estimated eight samples required per group. When three time points were considered (1, 2 and 4 weeks), we estimated in total to detect significant effects with seventy-two pigs.

For RNA sequencing analysis, six animals were used (three HZn $v$. three MZn), and this was done owing to monetary limitations, as we do not expect to reach a power of $80 \%$ at a significance level of 0.05 for testing differentially expressed genes directly from these data. Therefore, we used the data from RNA sequencing to perform GSEA. GSEA can be used to pick up general tendencies in the expression data and point to affected gene sets/pathways that are effected by $\mathrm{Zn}$ concentration (HZn $v$. MZn). Unfortunately, it is not possible to estimate the power obtained by GSEA; however, we control results at a false discovery rate of $5 \%$.

\section{Results}

\section{Expression of zinc transporters and zinc concentration} in gut-associated lymphatic tissue

The expression of $\mathrm{Zn}$ transporters is a prerequisite for the proper uptake of $\mathrm{Zn}$ ions by immune cells and immune systemassociated cells. We explored the repertoire of $\mathrm{Zn}$ transporters of GALT of piglets fed LZn, MZn and HZn diets for 1 and 2 weeks (Table 1 ). The expression of the long $Z I P 4$ transcripts ZIP4-201 and ZIP4-001 and the Zn transporters ZnT1, ZnT2 and $Z n T 5$ was measured in the MLN and Peyer's patches. The data provide evidence that ZIP4, ZnT1, ZnT2 and ZnT5 are expressed not only in the epithelium but also in the MLN and Peyer's patches of the jejunum (Table 1). However, there was no significant expression difference in these lymphoid tissues among the feeding groups for any examined $\mathrm{Zn}$ transporter gene, although we found a slight increase in $\mathrm{Zn}(14.31 \mathrm{mg} / \mathrm{kg})$ in the HZn group compared with MZn $(11.55 \mathrm{mg} / \mathrm{kg} ; P<0.05)$ and LZn $(12.45 \mathrm{mg} / \mathrm{kg} ; P<0 \cdot 1)$ groups in MLN after 1 week of feeding the $\mathrm{Zn}$ diets (Fig. 1). After 4 weeks of feeding, there was no difference in the $\mathrm{Zn}$ concentration for the feeding groups. 


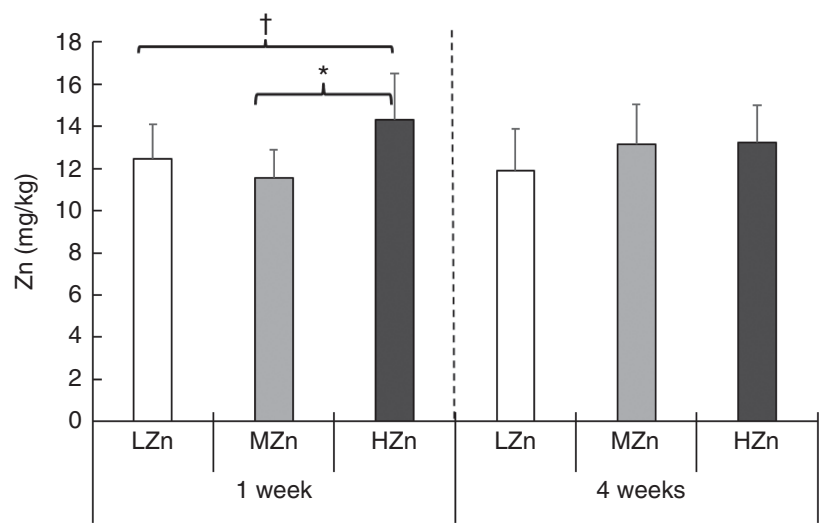

Fig. 1. Trace element concentration $(\mathrm{mg} / \mathrm{kg} \mathrm{DM})$ in mesenteric lymph nodes after 1 and 4 weeks of feeding a low zinc concentration of $57 \mathrm{mg} \mathrm{zinc} / \mathrm{kg}$ feed (LZn ( $\square$ ), 57 parts per million (ppm)), a medium zinc concentration of $164 \mathrm{mg}$ zinc/kg feed (MZn $(\square), 164 \mathrm{ppm}$ ) or a high zinc concentration of $2425 \mathrm{mg}$ zinc/ $\mathrm{kg}$ feed ( $\mathrm{HZn}(\square), 2425 \mathrm{ppm}$ ) to weaning piglets. Differences were tested using a pairwise Mann-Whitney $U$ test: ${ }^{*} 0.01<P<0.05, \dagger 0.05<P<0.1$.

\section{Short-term effects of zinc on T-cells}

After 1 week of feeding a HZn diet, piglets had a higher relative cell count of $\mathrm{CD}^{+}$and $\mathrm{CD} 8 \alpha^{\text {dim }}$ T-cells than piglets fed a diet with a LZn $(P<0.05)$ or MZn $(P<0 \cdot 01$; Fig. $2($ a $))$, suggesting that $\mathrm{Zn}$ feeding led to more activated status of T-helper cells. To be able to examine a direct effect of $\mathrm{Zn}$ ions on immune cells, we performed in vitro assays with primary isolated immune cells. In these in vitro assays, using isolated lymphocytes of MLN from a healthy donor pig, which was treated with different $\mathrm{Zn}$ concentrations $\left(0,10,100\right.$ and $\left.200 \mu \mathrm{M} \mathrm{Zn}^{2+}\right)$, we detected a tendency in the same direction, namely an elevated level of activated T-helper cells $\left(\mathrm{CD}^{+}\right.$and $\left.\mathrm{CD} 8 \alpha^{\mathrm{dim}}\right)$ after $3 \mathrm{~d}$ of culture in the HZn groups treated with 100 and $200 \mu \mathrm{M} \mathrm{Zn}^{2+}$ (Fig. 2(b)).

We next ascertained potential mechanisms responsible for the $\mathrm{Zn}$-associated increase in the level of activated T-helper cells at the transcript level using qPCR of relevant mediators. After 1 week of feeding the different $Z n$ diets, the T-helper cell 1-associated cytokine $I F N y$ was up-regulated at the transcript level in the HZn group when compared with the MZn $(P<0.05)$ and LZn $(P<0.05)$ groups within the GALT (Fig. 2(c)). Consistently with higher $I F N y$ transcript amounts, also higher mRNA levels of TBET, the master transcription factor of T-helper 1 cells, were observed in the HZn group compared with the LZn group (Fig. 2(c)) within the GALT $(P<0 \cdot 05)$. The higher transcript amount of $I F N \gamma$ was specifically seen within the ILLN (Fig. 2(c)). In addition, we determined expression levels of the T-helper cell-associated genes TBET, GATA3 and FOXP3 of lymphocytes from the cell culture-based experiment. After $3 \mathrm{~d}$ of treatment with $0,10,100$ or $200 \mu \mathrm{M} \mathrm{Zn}^{2+}$, we observed increased expression of TBET in lymphocytes treated with 100

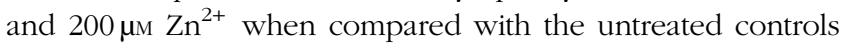
and the low-dose $\mathrm{Zn}$ treatment group of $10 \mu \mathrm{m} \mathrm{Zn}^{2+}$ (Fig. 2(d)) $(P<0.05)$. Hence, we could confirm the in vivo results in vitro. Within the cell culture-based experiment, we found a tendency of lower transcript amounts of FOXP3 in the 100 and $200 \mu \mathrm{M} \mathrm{Zn}^{2+}$ treatment groups compared with the control and the LZn 
(a)

In vivo effects of $\mathrm{Zn}$

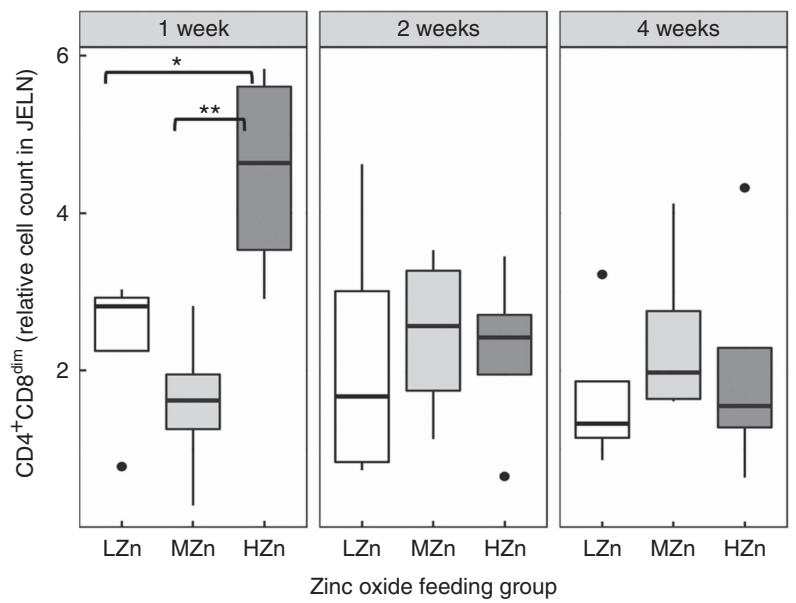

(c)

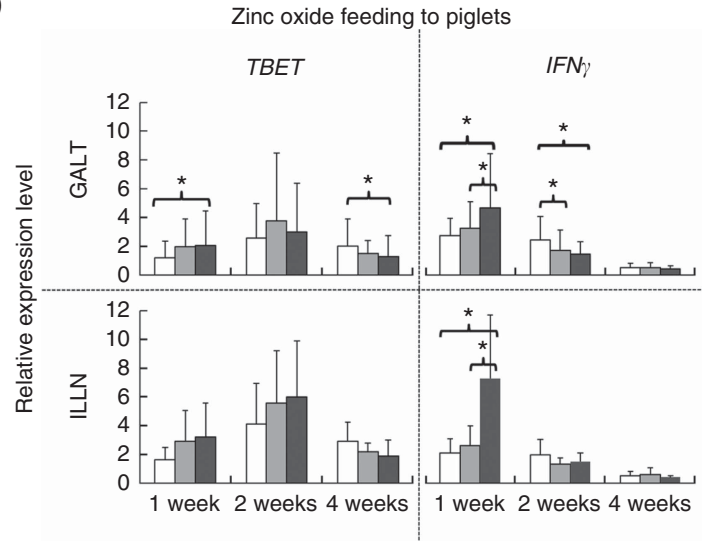

(b)

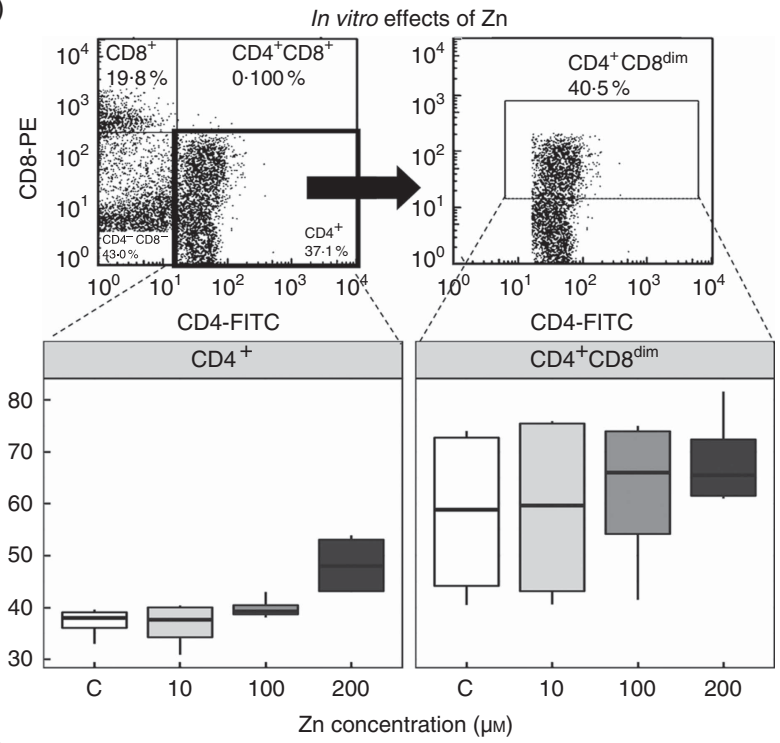

(d)

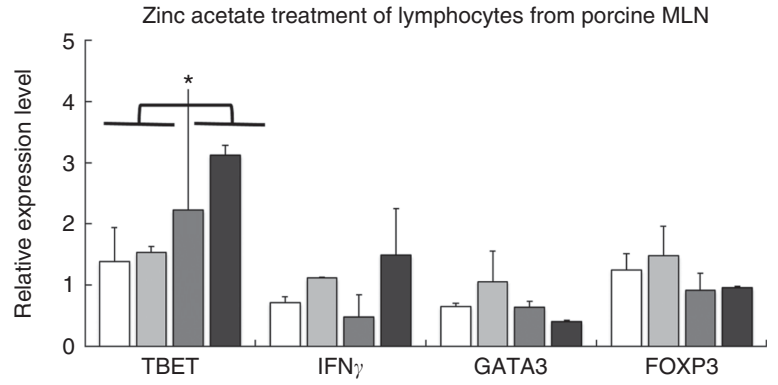

Fig. 2. (a) Cell counts relative to the living lymphocyte population of CD4 ${ }^{+} C D 8^{\text {dim }}$ T-helper cells of piglets fed low ( $57 \mathrm{mg}$ zinc/kg; LZn ( $\square$ )), medium (164 mg zinc/kg; MZn $(\square)$ ) or high (2425 mg zinc/kg; HZn $(\square)$ ) dosages, supplemented as zinc oxide for 1,2 or 4 weeks. (b) Cell counts relative to the living lymphocyte population of $\mathrm{CD}^{+}$and $\mathrm{CD} 4^{+} \mathrm{CD}^{\mathrm{dim}} \mathrm{T}$-helper cells of lymphocytes isolated from porcine mesenteric lymph nodes treated with 10,100 or $200 \mu \mathrm{m} \mathrm{Zn}^{2+} / \mathrm{well}^{\mathrm{ms}}$ zinc acetate for $3 \mathrm{~d}$ in vitro. (c) Relative mRNA expression of the master transcription factor for T-helper 1 cells T-box 21 (TBET) and the proinflammatory cytokine interferon $\gamma$ (IFN I $_{Y}$ in gutassociated lymphatic tissues (GALT) and ileocecal mesenteric lymph node (ILLN) of piglets fed zinc dosages as described in (a) for 1, 2 or 4 weeks. (d) Relative mRNA expression of the master transcription factors TBET, forkhead box P3 (FOXP3) and GATA binding protein 3 (GATA3), as well as the cytokine IFNy of lymphocytes isolated from porcine mesenteric lymph nodes (MLN) treated with 10,100, or $200 \mu \mathrm{M} \mathrm{Zn}^{2+} /$ well as zinc acetate for $3 \mathrm{~d}$ in vitro. Differences were tested using a pairwise

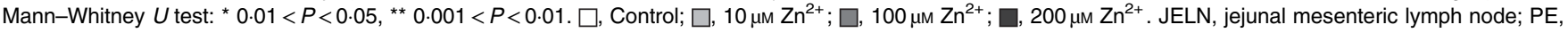
phycoerythrin; FITC, fluorescein isothiocyanate.

treatment group of $10 \mu \mathrm{m} \mathrm{Zn}^{2+}$ (Fig. 2(d)). GATA3 and FOXP3 showed no difference in expression after short-term feeding of zinc oxide (data not shown). In addition, we set up an in vitro experiment performed in a different laboratory using a different $\mathrm{Zn}$ source, zinc gluconate, to validate the found $\mathrm{Zn}$ effects on TBET within MLN. We also detected a higher expression of TBET 100 and $200 \mu \mathrm{M} \mathrm{Zn}^{2+}$ treatment groups compared with the control and the $\mathrm{LZn}$ treatment group of $10 \mu \mathrm{m} \mathrm{Zn}^{2+}$ (online Supplementary Fig. S1). Furthermore, we checked the response of lymphocytes derived from blood within the in vitro assay using zinc gluconate. We could not detect a difference in TBET expression for lymphocytes from blood. In a next step, we were interested in the expression of important T-regulating genes and choose IL12 and PKCtheta. Remarkably, PKCtheta was highly up-regulated with $\mathrm{Zn}$ treatment in MLN-derived cells, whereas the up-regulation in blood-derived immune cells was moderate. IL12 showed an increase of expression in lymphocytes derived from MLN and from blood in the HZn treatment groups.

To better understand the mechanism, accounting for the short-term effects of high dietary $\mathrm{Zn}$, we performed RNA sequencing of MLN from 32-d-old piglets after feeding MZn $(\mathrm{MZn}=$ three individual piglets) and $\mathrm{HZn}$ concentration ( $\mathrm{HZn}=$ three individual piglets) in the feed for 1 week. To get a picture of contributing pathways, we performed GSEA on our differentially expressed genes. GSEA revealed highest enrichment for genes associated with cytokine signalling in the immune system (normalised enrichment score $=2 \cdot 22$ ) (Fig. 3). In addition, GSEA identified a high over-representation of pathways involved in innate and adaptive immune response, within our differentially expressed genes, showing a massive activation of immune response in the HZn group compared with the MZn group after 1 week of feeding the zinc oxide diets. 


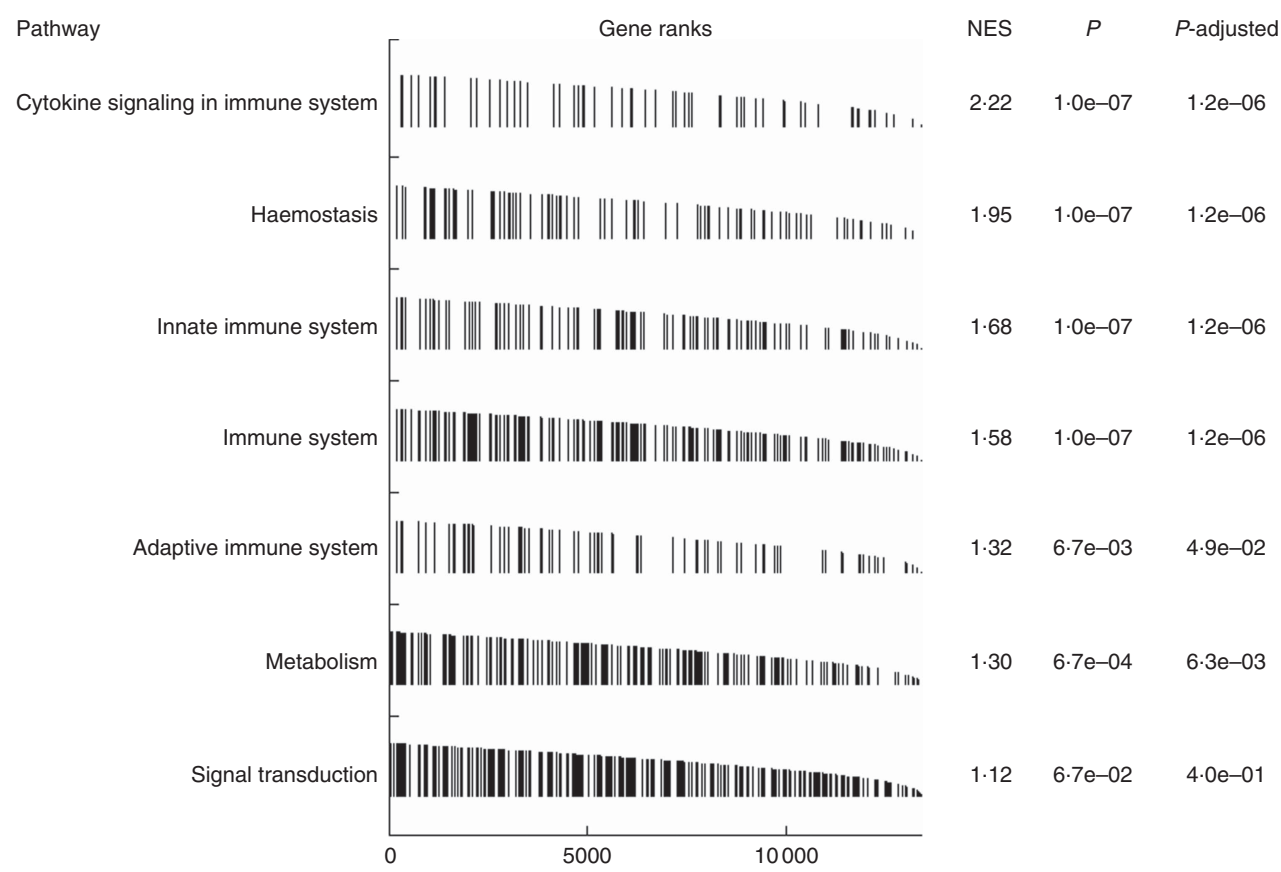

Fig. 3. Gene set enrichment analysis on differentially expressed genes within ileocecal mesenteric lymph node tissue between the feeding groups medium zinc concentration (164 mg zinc/kg feed) and high zinc concentration ( $2425 \mathrm{mg} \mathrm{zinc/kg}$ feed) after 1 week of feeding ranked to the normalised enrichment score (NES) from high to low.

\section{Mid- and long-term effects of feeding zinc oxide}

After 2 weeks of feeding the different Zn diets, the HZn group had higher transcript amounts of FOXP3, the master transcription factor of regulatory T-cells ${ }^{(27)}$, in the HZn group compared with the MZn $(P<0.01)$ and LZn $(P<0.05)$ groups within the GALT (Fig. 4(a)). In particular, we observed a higher expression of FOXP3 in JELN $(P<0.05)$ and JEPP $(P<0.05)$, as well as in ILLN $(P<0.05)$, in the HZn feeding group when compared to the LZn and MZn groups after 2 weeks of feeding the $\mathrm{Zn}$ diets (Fig. 4(b)).

We confirmed increased frequencies of $\mathrm{CD}^{+} \mathrm{CD} 25^{\text {high }}$ regulatory T-cells among ILLN and JEPP and ILPP, respectively, in the HZn group compared with the MZn group $(P<0.05)$ by phenotypic analysis of immune cells using FCM (Fig. 4(c) and (d)). Moreover, JELN and PAPIL showed comparable tendencies in the same direction, which is a higher relative cell count of $\mathrm{CD}^{+} \mathrm{CD} 25^{\text {high }}$ regulatory $\mathrm{T}$-cells in the $\mathrm{HZn}$ group when compared with the MZn Zn feeding group $(P<0 \cdot 1)$.

After long-term feeding of 4 weeks of the zinc oxide diets, we observed lower relative cell counts of $\mathrm{CD}^{+}{ }^{+} \mathrm{T}$-helper cells in the HZn group when compared with the MZn group for jejunal lymph nodes $(P<0.05)$ (Fig. 5). We additionally found a tendency for a lower relative cell count of $\mathrm{CD}^{+}$ cytotoxic T-cells in the HZn group compared with the MZn group $(P<0 \cdot 1)$ after 4 weeks of feeding the $\mathrm{Zn}$ diets. The $\mathrm{LZn}$ group showed a reduction in relative cell counts of $\mathrm{CD}^{+}$ cytotoxic T-cells $(P<0.05)$ compared with our control group fed a MZn diet (Fig. 5). The same tendencies could be seen in ileal lymph nodes (data not shown). In addition, we detected a lower relative transcript amount of TBET within GALT (Fig. 2(c)).

\section{Discussion}

Our result shows that immune cells derived from MLN and Peyer's patches showed expression of ZIP4, ZnT1, ZnT2 and $Z n T 5$ in all feeding regimens. To our knowledge, there is currently no information available that shows expression of $\mathrm{Zn}$ transporters in porcine immune cells. There is vast literature available linking $\mathrm{Zn}$ with immune function, as shown in the review paper by Wessels et al. ${ }^{(28)}$. Our hypothesis was that $\mathrm{Zn}$ transporters on the surface of immune cells respond to differences in $\mathrm{Zn}$ concentration. To validate this hypothesis, mRNA expression levels of the most common porcine $\mathrm{Zn}$ transporters (ZIP4, ZnT1, ZnT2 and ZnT5) were analysed. These genes were selected on the basis of previous research $^{(29,30)}$, which showed differential expression of these transporters in epithelial cells and pancreatic cancer cells. However, in our current study, no differential expression of the selected Zn transporters was detected (Table 1).

As expected, a significantly higher $\mathrm{Zn}$ concentration was detected in MLN of the HZn feeding group compared with the MZn group after 1 week $(P<0 \cdot 05)$ of feeding. After 4 weeks, no difference in $\mathrm{Zn}$ concentration was observed (Fig. 1). Zn concentrations in MLN were surprisingly low compared with other tissues (jejunum, liver and pancreas) ${ }^{(17)}$. The low total $\mathrm{Zn}$ concentration in MLN, as well as the marginal changes in this concentration after feeding high levels of zinc oxide, indicates that MLN tightly control intra-cellular Zn levels. However, as no differential expression of $\mathrm{Zn}$ transporters was detected, the mode of regulation remains elusive.

Investigation of time-dependent, Zn-associated effects on adaptive immune cells upon HZn feeding showed that shortterm feeding (1 week) led to significantly higher numbers of 

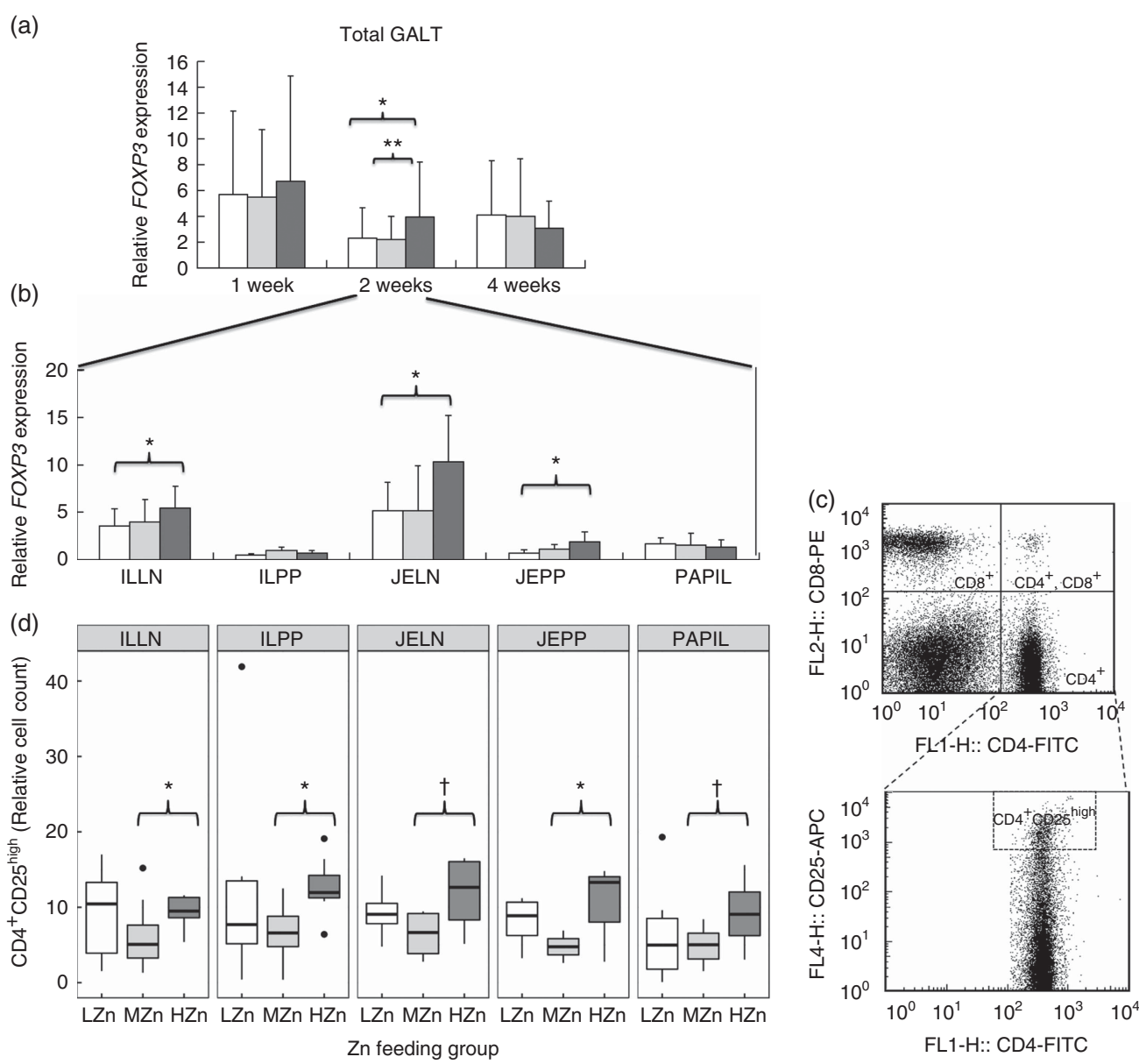

Fig. 4. (a and b) Relative mRNA expression of forkhead box P3 (FOXP3) in gut-associated lymphatic tissue (GALT) and in jejunal and ileocecal mesenteric lymph nodes (JELN and ILLN, respectively), Peyer's patches from the jejunum and the ileum (JEPP and ILPP, respectively) and ileal papilla (PAPIL) analysed separately. (c) Exemplary original flow plot showing used gates to analyse cell populations. Plots are done using FlowJo version 7.9. displaying CD8-phycoerythrin (PE) against CD4fluorescein isothiocyanate (FITC) staining and CD25-allophycocyanin (APC) against CD4-FITC. (d) Cell counts, relative to the living lymphocyte population of $\mathrm{CD} 4^{+} \mathrm{CD} 25^{\text {high }}$ regulatory T-helper cells shown for the same tissues as in (b). Differences were tested using a pairwise Mann-Whitney $U$ test: ${ }^{*} 0.01<P<0.05$, ${ }^{*}$ $0.001<P<0.01, \dagger 0.05<P<0.1$. $\square$, Low zinc concentration (57 parts per million (ppm)); $\square$, medium zinc concentration (164 ppm) and $\square$, high zinc concentration (2425 ppm)
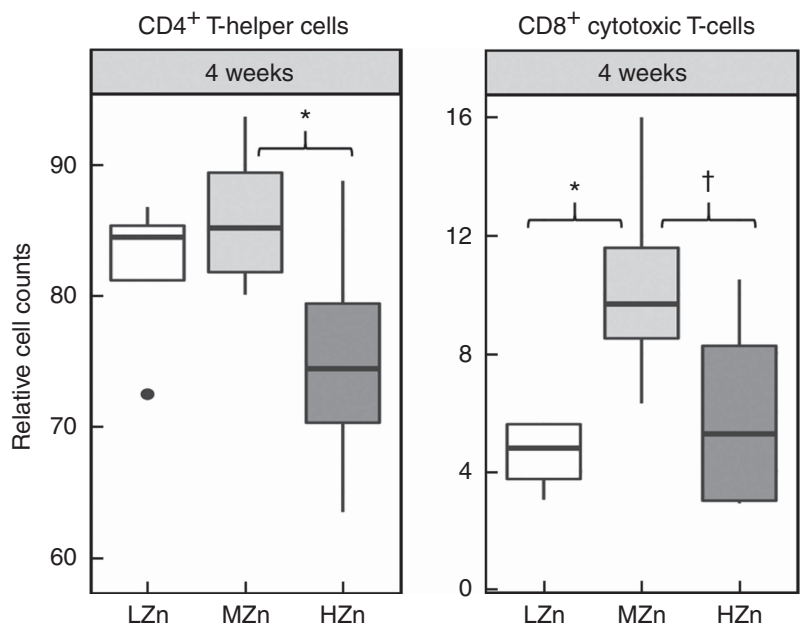

Fig. 5. Cell counts relative to the living lymphocyte population of $C D 4^{+} T$-helper cells and $\mathrm{CD} \mathrm{b}^{+}$cytotoxic T-cells of jejunal mesenteric lymph nodes from piglets fed low zinc (57 mg zinc/kg) in diet (LZn), $164 \mathrm{mg}$ zinc/kg (MZn) or $2425 \mathrm{mg}$ zinc $/ \mathrm{kg}(\mathrm{HZn})$ as zinc oxide for 4 weeks. Differences were tested using a pairwise Mann-Whitney $U$ test: ${ }^{*} 0.01<P<0.05, \dagger 0.05<P<0.1$. activated T-helper cells $\left(\mathrm{CD} 4^{+}\right.$and $\left.\mathrm{CD} 8 \alpha^{\mathrm{dim}}\right)(P<0 \cdot 01)$. Molecular analysis revealed higher transcript amounts of $I F N \gamma$ and TBET in the HZn group compared with the LZn group $(P<0.05)$. TBET is the master transcription factor of T-helper 1 cells, whereas $I F N \gamma$ is considered the main inducer of T-helper 1 cells $^{(31)}$. Our work provides support for the hypothesis that the differentiation of naive T-helper cells towards T-helper 1 cells might be short-term inducible by high levels of dietary $\mathrm{Zn}$. In addition, a tendency was detected showing a higher relative cell count of cytotoxic T-cells within the HZn group compared with the MZn group after 1 week $(P<0 \cdot 1$, data not shown).

GSEA on differentially expressed genes between MZn and $\mathrm{HZn}$ groups showed an over-representation of genes belonging to the pathway 'cytokine signalling in immune system'. This points towards an activation of the immune system after a 1-week feeding of zinc oxide.

To investigate this effect further, in vitro experiments can be used as a model to investigate direct effects of $\mathrm{Zn}$ on the immune cells. These in vitro experiments in which porcine MLN were treated with zinc acetate (Fig. 2), as well as zinc 
gluconate (online Supplementary Fig. S1), replicated the in vivo results: a tendency towards higher relative cell counts of activated T-helper cells and a higher transcript level of TBET. As we used zinc acetate, as well as zinc gluconate, within the in vitro assays and zinc oxide in the feeding experiment, this effect is very likely related to the $\mathrm{Zn}$ ion itself and not due to the specific oxide. Similar findings were reported by Barnett et $a l .{ }^{(32)}$ who showed that an increase in serum $\mathrm{Zn}$ concentrations was associated with an increase in the number of T-cells in elderly people supplemented with $30 \mathrm{mg} \mathrm{Zn} / \mathrm{d}$ in the form of zinc gluconate.

After 2 weeks of feeding HZn diets, there seem to be a switch of the immune response from an immune activation towards suppression. Higher relative cell counts of immune-suppressive $\mathrm{CD} 4^{+} \mathrm{CD} 25^{\text {high }}$ regulatory T-helper cells were detected in all five analysed GALT. This was accompanied with higher transcript amounts of the FOXP3 gene, the master transcription factor of regulatory T-helper cells in the HZn-group compared to the MZn-group. Regulatory T-helper cells have a suppressive function on the inflammatory immune response ${ }^{(33)}$ and are reported to be capable of suppressing proliferation and differentiation of effector T-cells ${ }^{(34)}$. As suggested by Pandiyan et $a l .{ }^{(35)}$, this could be owing to induced Bcl-2-like protein 11mediated apoptosis of activated effector $\mathrm{CD}^{+}$T-cells through the uptake of $I L-2$. In line with previous result, our results showed a reduction in the relative $\mathrm{CD}^{+}{ }^{+} \mathrm{T}$-cell count and also a tendency towards reduced relative cell counts of cytotoxic Tcells after 4 weeks in the HZn group compared with the MZn group. This is probably owing to the higher levels of regulatory T-helper cells detected after 2 weeks of feeding an HZn diet.

$\mathrm{Zn}$ taken orally is considered to be relatively nontoxic. However, toxicity symptoms have been known to occur with high levels of $\mathrm{Zn}$ intake in humans ${ }^{(36)}$ (approximately 100$300 \mathrm{mg} \mathrm{Zn/d}$ ) when $\mathrm{Zn}$ is taken for longer periods of time (>10 months) $)^{(37)}$. Long-term $\mathrm{Zn}$ toxicity is often accompanied by a severe $\mathrm{Cu}$ deficiency (hypocupraemia); after cessation of the use of $\mathrm{Zn}$ supplements, the $\mathrm{Cu}$ levels often normalise ${ }^{(38)}$. It is believed that the excess of $\mathrm{Zn}$ blocks the intestinal uptake of $\mathrm{Cu}$, leading to hypocupraemia, until the excess of $\mathrm{Zn}$ is eliminated from the system. High levels of $\mathrm{Zn}$ intake $(300 \mathrm{mg}$ $\mathrm{Zn} / \mathrm{d}$ ) in humans for longer periods of time ( 6 weeks) have also shown to depress indices of immune function in healthy adults compared with baseline levels before supplementation ${ }^{(15)}$. However, this effect was not seen in an elderly population exposed to lower levels of supplementation $(100 \mathrm{mg} \mathrm{Zn/d})^{(39)}$.

In a previous study with a similar experimental setup, we observed after 6 weeks feeding of HZn levels (2500 mg Zn/kg as zinc oxide) a decreased relative cell count of $\mathrm{CD}^{+} \mathrm{T}$ and natural killer cells, CD8 $\mathrm{a}^{\text {high }}$ cytotoxic T-cells and $\mathrm{CD} 4^{+} \mathrm{T}$-helper cells in MLN comparable to the situation in the LZn feeding group $^{(13)}$. This indicates a T-cell suppressive effect for both $\mathrm{Zn}$ deficiency and $\mathrm{Zn}$ excess. Interestingly, weight gain in the HZn group was only observed during the first week, but the weight gain was lower by the third week of the experiment compared with optimal- and low-level $\mathrm{Zn}$ diets ${ }^{(23)}$, suggesting an optimal feeding time of 2 weeks when feeding high levels of zinc oxide.

In summary, our research suggests that short-term feeding (1-2 weeks) of high levels of $\mathrm{Zn}$ at the critical time point of weaning has an immune-activating effect on the adaptive T-cell response and could provide an alternative to reduce the incidence of post-weaning diarrhoea in pig husbandry. However, our findings indicate that long-term feeding (2-4 weeks) of high levels of $\mathrm{Zn}$ seems to have an immune-suppressive effect in post-weaned piglets and as such could potentially negate the benefits of $\mathrm{Zn}$ supplements in post-weaned piglets.

\section{Acknowledgements}

The study was funded by the German Research Foundation (Deutsche Forschungsgemeinschaft, DFG) within the Collaborative Research Group (SFB, Sonderforschungsbereich) 852/1 'Nutrition and intestinal microbiota - host interactions in the pig'. The authors are solely responsible for the data and do not represent any opinion of neither the DFG nor other public or commercial entity.

S. K.-R., J. N. S. and D. K. performed the experiments; D. A., P. K. and S. K.-R. analysed the data; R. P., F. M. and V. G. contributed reagents/materials/analysis tools; J. Z. and G. A. B. conceived and designed the experiments and S. K.-R. and D. A. wrote the paper.

The authors declare no conflict of interest. The founding sponsors had no role in the design of the study; in the collection, analyses or interpretation of data; in the writing of the manuscript; and in the decision to publish the results.

\section{Supplementary material}

For supplementary material/s referred to in this article, please visit https://doi.org/10.1017/S0007114518002908

\section{References}

1. Sharma A, Patni B, Shankhdhar D, et al. (2013) Zinc - an indispensable micronutrient. Physiol Mol Biol Plants 19, 11-20.

2. World Health Organization (2013) Zinc supplementation and growth in children: biological, behavioural and contextual rationale. e-Library of Evidence for Nutrition Actions (eLENA). http://www.who.int/elena/titles/bbc/zinc_stunting/en/

3. Penny ME (2013) Zinc supplementation in public health. Ann Nutr Metab 62, Suppl. 1, 31-42.

4. Kim J \& Ahn J (2014) Effect of zinc supplementation on inflammatory markers and adipokines in young obese women. Biol Trace Elem Res 157, 101-106.

5. Solomons NW (2013) Update on zinc biology. Ann Nutr Metab 62, Suppl. 1, 8-17.

6. Prasad AS (2000) Effects of zinc deficiency on Th1 and Th2 cytokine shifts. J Infect Dis 182, Suppl. 1, S62-S68.

7. Kim J, Ahn H, Woo HM, et al. (2014) Characterization of porcine NLRP3 inflammasome activation and its upstream mechanism. Vet Res Commun 38, 193-200.

8. Meurens F, Summerfield A, Nauwynck H, et al. (2012) The pig: a model for human infectious diseases. Trends Microbiol 20, 50-57.

9. Molist F, Hermes RG, de Segura AG, et al. (2011) Effect and interaction between wheat bran and zinc oxide on productive performance and intestinal health in post-weaning piglets. $\mathrm{BrJ}$ Nutr 105, 1592-1600.

10. Pieper R, Vahjen W, Neumann K, et al. (2012) Dosedependent effects of dietary zinc oxide on bacterial 
communities and metabolic profiles in the ileum of weaned pigs. J Anim Physiol Anim Nutr (Berl) 96, 825-833.

11. Sales J (2013) Effects of pharmacological concentrations of dietary zinc oxide on growth of post-weaning pigs: a metaanalysis. Biol Trace Elem Res 152, 343-349.

12. Shen J, Chen Y, Wang Z, et al. (2014) Coated zinc oxide improves intestinal immunity function and regulates microbiota composition in weaned piglets. Br J Nutr 111, 2123-2134.

13. Janczyk P, Kreuzer S, Assmus J, et al. (2013) No protective effects of high-dosage dietary zinc oxide on weaned pigs infected with Salmonella enterica serovar Typhimurium DT104. Appl Environ Microbiol 79, 2914-2921.

14. Schulte JN, Brockmann GA \& Kreuzer-Redmer S (2016) Feeding a high dosage of zinc oxide affects suppressor of cytokine gene expression in Salmonella Typhimurium infected piglets. Vet Immunol Immunopathol 178, 10-13.

15. Chandra RK (1984) Excessive intake of zinc impairs immune responses. JAMA 252, 1443-1446.

16. Han YK, Hwan Hwang IL \& Thacker PA (2011) Use of a micro-encapsulated eucalyptus-medium chain fatty acid product as an alternative to zinc oxide and antibiotics for weaned pigs. J Swine Health Prod 19, 34-43.

17. Zetzsche A, Schunter N, Zentek J, et al. (2016) Accumulation of copper in the kidney of pigs fed high dietary zinc is due to metallothionein expression with minor effects on genes involved in copper metabolism. J Trace Elem Med Biol 35, 1-6.

18. Liu P, Pieper R, Tedin L, et al. (2014) Effect of dietary zinc oxide on jejunal morphological and immunological characteristics in weaned piglets. J Anim Sci 92, 5009-5018.

19. Karweina D, Kreuzer-Redmer S, Müller U, et al. (2015) The zinc concentration in the diet and the length of the feeding period affect the methylation status of the ZIP4 zinc transporter gene in piglets. PLOS ONE 10, e0143098.

20. Kroger S, Pieper R, Aschenbach JR, et al. (2015) Effects of high levels of dietary zinc oxide on ex vivo epithelial histamine response and investigations on histamine receptor action in the proximal colon of weaned piglets. J Anim Sci $\mathbf{9 3}$, $5265-5272$

21. Chai W, Zakrzewski SS, Gunzel D, et al. (2014) High-dose dietary zinc oxide mitigates infection with transmissible gastroenteritis virus in piglets. BMC Vet Res 10, 75.

22. Brugger D \& Windisch WM (2016) Subclinical zinc deficiency impairs pancreatic digestive enzyme activity and digestive capacity of weaned piglets. Br J Nutr 116, 425-433.

23. Martin L, Pieper R, Schunter N, et al. (2013) Performance, organ zinc concentration, jejunal brush border membrane enzyme activities and mRNA expression in piglets fed with different levels of dietary zinc. Arch Anim Nutr 67, 248-261.

24. Kreuzer S, Janczyk P, Assmus J, et al. (2012) No beneficial effects evident for Enterococcus faecium NCIMB 10415 in weaned pigs infected with Salmonella enterica serovar Typhimurium DT104. Appl Environ Microbiol 78, 4816-4825.

25. Kreuzer S, Machnowska P, Assmus J, et al. (2012) Feeding of the probiotic bacterium Enterococcus faecium NCIMB 10415 differentially affects shedding of enteric viruses in pigs. Vet Res 43, 58.

26. Livak KJ \& Schmittgen TD (2001) Analysis of relative gene expression data using real-time quantitative PCR and the 2 (-Delta Delta C(T)) Method. Methods 25, 402-408.

27. Fontenot JD, Gavin MA \& Rudensky AY (2003) Foxp3 programs the development and function of $\mathrm{CD}^{+} \mathrm{CD} 25^{+}$regulatory T cells. Nat Immunol 4, 330-336.

28. Wessels I, Maywald M \& Rink L (2017) Zinc as a gatekeeper of immune function. Nutrients $\mathbf{9}, 1286$.

29. Jayaraman AK \& Jayaraman S (2011) Increased level of exogenous zinc induces cytotoxicity and up-regulates the expression of the ZnT-1 zinc transporter gene in pancreatic cancer cells. J Nutr Biochem 22, 79-88.

30. Martin L, Lodemann U, Bondzio A, et al. (2013) A high level of dietary zinc changes the expression of zinc transporters and metallothionein in vitro and in the jejunum, but does not prevent zinc accumulation in jejunal tissue of piglets. $J$ Nutr 143, 1205-1210.

31. Oestreich KJ \& Weinmann AS (2012) Transcriptional mechanisms that regulate T helper 1 cell differentiation. Curr Opin Immunol 24, 191-195.

32. Barnett JB, Dao MC, Hamer DH, et al. (2016) Effect of zinc supplementation on serum zinc concentration and $\mathrm{T}$ cell proliferation in nursing home elderly: a randomized, doubleblind, placebo-controlled trial. Am J Clin Nutr 103, 942-951.

33. Miyao T, Floess S, Setoguchi R, et al. (2012) Plasticity of Foxp $3^{+} \mathrm{T}$ cells reflects promiscuous Foxp3 expression in conventional $\mathrm{T}$ cells but not reprogramming of regulatory T cells. Immunity 36, 262-275.

34. Letourneau S, Krieg C, Pantaleo G, et al. (2009) IL-2- and CD25-dependent immunoregulatory mechanisms in the homeostasis of T-cell subsets. J Allergy Clin Immunol $\mathbf{1 2 3}$, 758-762.

35. Pandiyan P, Zheng L, Ishihara S, et al. (2007) $\mathrm{CD}^{+} \mathrm{CD} 25^{+} \mathrm{Foxp}^{+}$regulatory $\mathrm{T}$ cells induce cytokine deprivation-mediated apoptosis of effector $\mathrm{CD}^{+}{ }^{+} \mathrm{T}$ cells. Nat Immunol 8, 1353-1362.

36. Fosmire GJ (1990) Zinc toxicity. Am J Clin Nutr 51, 225-227.

37. Murphy JV (1970) Intoxication following ingestion of elemental zinc. JAMA 212, 2119-2120.

38. Prasad AS, Brewer GJ, Schoomaker EB, et al. (1978) Hypocupremia induced by zinc therapy in adults. JAMA $\mathbf{2 4 0}$, 2166-2168.

39. Bogden JD, Oleske JM, Lavenhar MA, et al. (1988) Zinc and immunocompetence in elderly people: effects of zinc supplementation for 3 months. Am J Clin Nutr 48, 655-663. 